\title{
AMETROPIA AND SEX
}

\author{
BY \\ J. A. WILSON \\ CAMBUSLANG, GLASGOW
}

IN Lanarkshire during a period of eight years over 22,000 school children with defective vision were sent to the various treatment centres. . Of these children 57.9 per cent. were girls. At one of these treatment centres the cases were divided into two groups, (1) the hypermetropia group, consisting of cases of hypermetropia and cases of hypermetropic astigmatism, and (2) the myopia group, consisting of cases of myopia, myopic astigmatism and mixed astigmatism.

They were also arranged in age-groups, (1) under seven years, (2) seven years to ten years inclusive, and (3) eleven to fourteen years.

Age-group under seven years.-From among the scholars who have been examined during the last eight years I have picked out all those under seven years of age. This gave me a group of 300 children and in this group there are eight cases of hypermetropia to one of myopia and the boys and girls are equal in number. Among the cases in the hypermetropia group there are some with $8 \mathrm{D}$. of hypermetropia and in the myopia group there is one with 12 D. of myopia. Between these there are many intermediate conditions. In the myopia group 12 had low, 8 medium and 15 high degrees of myopia. There are also 14 cases that may be said to be on the border line.

Most of the work these children get to do in the school is reading or observing at a distance of several yards, and in their little school-books the type is large.

School-work, in my opinion, is not responsible for these cases of myopia.

Age-group 7 to 10 years. - In this group there are 4.6 cases of hypermetropia to one of myopia and the percentage of girls is 55 . These estimates are based on 800 consecutive cases.

Age-group 11 to 14 years. - In this age-group there are 3 cases of hypermetropia to one of myopia and the percentage of girls has risen to 59 . These estimates are based on 1,200 consecutive cases. Quite a large number of people become myopic at and beyond puberty and therefore the ultimate percentage of females is higher still.

Conditions in Higher Grade pupils.-During the last eight years 94 pupils from Higher Grade schools have been examined. Their ages range from 15 to 17 years; 53 were males and 41 
females. Here the approximation between the hypermetropia and myopia groups has now reached parity, for one half of these pupils were found to be myopic. These relative proportions are striking, but the number of cases is small. The results may or may not have some relation to the amount of study associated with these higher grade schools. Myopic people are often mentally equipped for higher-grade work and their personal inclinations frequently lie in the same direction. Thus there may be selective influences at work in this group.

Some years ago I collected 1,500 consecutive cases of myopia and investigated their family histories. These were hospital cases and were of all ages. The task occupied two years. It was observed that where a parent, father or mother, was myopic then that parent transmitted myopia to two girls for one bov. This is not always obvious in individual families, but it is obvious when families are taken collectively.

These cases are not quite comparable with the school percentages, but nevertheless the school figures move steadily towards this ratio of two girls for one boy. Both sets of figures seem to be parts of one and the same process, namely the operation of a law of inheritance that does not become fully manifest till we reach adult life.

\section{INVITATION MEETING OF THE BELGIAN OPHTHALMOLOGICAL SOCIETY}

THE Belgian Ophthalmological Society invited a number of guests from adjoining countries to a special Congress during the continuance of the great International Exhibition at Brussels this year. The meetings were held on the 21st, 22nd and 23rd September, and among the guests were representatives from France, Holland, Spain, Switzerland and Great Britain. The scientific business was conducted in the new hospital of Saint-Pierre, which from now onwards will replace the old hospital of Saint-Jean as the Medical School of the University.

Dr. M. Danis, who succeeds Prof. Coppez as Professor of Ophthalmology in the University of Brussels, presided over the meetings and we congratulate him heartily on the equipment of his new ophthalmic department at Saint-Pierre.

On Saturday afternoon (21st) we had two lectures, one by a Physician, Dr. Paul Govaerts, Professor of Medicine in the University, on the physiopathology of kidney inflammations, the 